\title{
Treatment of ventilator-associated pneumonia (VAP) caused by Acinetobacter: results of prospective and multicenter ID-IRI study
}

\author{
Hakan Erdem ${ }^{1}$ (D) $\cdot{\text { Yasemin } \mathrm{Cag}^{2} \cdot \text { Serap Gencer }}^{3} \cdot$ Serhat Uysal $^{4} \cdot$ Zuhal Karakurt $^{5} \cdot$ Rezan Harman $^{6} \cdot$ Emel Aslan $^{7}$. \\ Esmeray Mutlu-Yilmaz ${ }^{8}$. Oguz Karabay ${ }^{9}$ - Yesim Uygun ${ }^{10} \cdot$ Mehmet Ulug $^{11}$. Selma Tosun ${ }^{12} \cdot$ Arzu Dogru $^{2}$. \\ Alper Sener ${ }^{13} \cdot$ Mustafa Dogan $^{14} \cdot$ Rodrigo Hasbun $^{15} \cdot$ Gul Durmus $^{16} \cdot$ Hale Turan $^{17}$. Ayse Batirel ${ }^{18} \cdot$ Fazilet Duygu $^{19}$. \\ Asuman Inan ${ }^{20}$. Yasemin Akkoyunlu ${ }^{21} \cdot$ Guven Celebi $^{22} \cdot$ Gulden Ersoz $^{23} \cdot$ Tumer Guven $^{24} \cdot$ Ozgur Dagli $^{16}$. \\ Selma Guler ${ }^{25} \cdot$ Meliha Meric-Koc $^{21} \cdot$ Serkan Oncu ${ }^{26} \cdot$ Jordi Rello ${ }^{27}$
}

Received: 4 July 2019 / Accepted: 26 August 2019 / Published online: 9 September 2019

(C) Springer-Verlag GmbH Germany, part of Springer Nature 2019

\begin{abstract}
Ventilator-associated pneumonia (VAP) due to Acinetobacter spp. is one of the most common infections in the intensive care unit. Hence, we performed this prospective-observational multicenter study, and described the course and outcome of the disease. This study was performed in 24 centers between January 06, 2014, and December 02, 2016. The patients were evaluated at time of pneumonia diagnosis, when culture results were available, and at $72 \mathrm{~h}$, at the 7th day, and finally at the 28th day of follow-up. Patients with coexistent infections were excluded and only those with a first VAP episode were enrolled. Logistic regression analysis was performed. A total of 177 patients were included; empiric antimicrobial therapy was appropriate (when the patient received at least one antibiotic that the infecting strain was ultimately shown to be susceptible) in only 69 (39\%) patients. During the 28-day period, antibiotics were modified for side effects in $27(15.2 \%)$ patients and renal dose adjustment was made in 38 (21.5\%). Ultimately, 89 (50.3\%) patients died. Predictors of mortality were creatinine level (OR, 1.84 (95\% CI 1.279-2.657); $p=$ 0.001), fever (OR, 0.663 (95\% CI 0.454-0.967); $p=0.033$ ), malignancy (OR, 7.095 (95\% CI 2.142-23.500); $p=0.001$ ), congestive heart failure (OR, 2.341 (95\% CI 1.046-5.239); $p=0.038$ ), appropriate empiric antimicrobial treatment (OR, 0.445 (95\% CI 0.216-0.914); $p=0.027$ ), and surgery in the last month (OR, 0.137 (95\% CI 0.037-0.499); $p=0.003$ ). Appropriate empiric antimicrobial treatment in VAP due to Acinetobacter spp. was associated with survival while renal injury and comorbid conditions increased mortality. Hence, early diagnosis and appropriate antibiotic therapy remain crucial to improve outcomes.
\end{abstract}

Keywords Ventilator-associated pneumonia $\cdot$ VAP $\cdot$ Pneumonia $\cdot$ Acinetobacter $\cdot$ Mortality $\cdot$ Treatment

\section{Introduction}

Ventilator-associated pneumonia (VAP) is one of the most common infections in the intensive care unit (ICU) [1,2]. The disease represents a diagnostic and management dilemma to clinicians [3] and is associated with significant mortality in patients [4]. Acinetobacter strains, once considered a lowcategory pathogen, have become an important etiology of VAP in ICUs worldwide [5, 6]. Patients with serious comorbidities that need mechanical ventilation can become infected

Hakan Erdem

hakanerdem1969@yahoo.com

Extended author information available on the last page of the article with antibiotic-resistant Acinetobacter spp., representing a therapeutic challenge [7,8]. There is a paucity of information on VAP due to Acinetobacter spp. Thus, we performed this prospective-observational multicenter study, and described the course, prognostic factors, and outcomes of VAP due to Acinetobacter spp.

\section{Methods}

An observational, prospective study was performed in 24 medical centers between January 06, 2014, and December 02, 2016. Dr. Lutfi Kirdar Education and Training Hospital's Review Board in Istanbul approved the study (02/01/2014VIP 2014/1) and this approval was confirmed by the Turkish 
Ministry of Health, Drugs and Pharmaceutics Agency for all participating centers. This study was performed in accordance with the Helsinki Declaration of 1964 and its later amendments.

Appropriate empiric antimicrobial treatment was defined if the patient received at least one antibiotic that the infecting strain was ultimately shown to be susceptible. The laboratories of the participant centers used Vitek-2 $(n=20)$, BD Phoenix M50 $(n=2)$, broth microdilution $(n=1)$, and Microscan $96(n=1)$ for antibiotic susceptibility testing in the participating centers in accordance with EUCAST guidelines during the study period [9-11]. In addition, E-test was used as a complementary method to Vitek-2 in three centers. Multidrug resistance (MDR), extensively drug resistance (XDR), and pan-drug resistance (PDR) were classified according to definitions elsewhere [12].

\section{Data collection and procedures}

The same questionnaire was used throughout all participant centers and the data input was made available to centers through the internet. The patients enrolled in the study were regularly evaluated by the consulting infectious disease physician at the time of pneumonia suspicion/diagnosis, when antibiotic susceptibility testing (AST) results were available, at $72 \mathrm{~h}$, at the 7 th day, and finally at the 28th day of follow-up. All antibiotics were prescribed by an infectious disease physician. The decision to start or modify antimicrobial chemotherapy was made by infectious disease clinicians. There was no policy for clinicians to choose antimicrobials in the study protocol.

\section{Inclusion criteria}

1. Age $\geq 18$ years

2. Patients mechanically ventilated $(>48 \mathrm{~h})$

3. Pulmonary infection due to Acinetobacter spp. as the first episode

4. Presence of systemic, radiological, clinical/pulmonary, and microbiological findings indicating VAP [13]

5. Empirical antibiotic therapy should have been started

6. Written consent should be obtained either from the patient or from her/his close relatives

\section{Exclusion criteria}

1. Pregnancy

2. The presence of concordant/coexistent infection other than VAP

3. Presence of an infection detected preceding VAP or if the patient was still given antibiotics at the time of VAP diagnosis
4. The recovery of other bacteria along with the infecting Acinetobacter spp. either in blood or in bronchial samples

5. If qualitative cultures of respiratory specimens were done solely

Missing data Cases with missing and/or outliers were asked to be corrected by the researcher of the center. Variables with more than $30 \%$ missing value between all candidate predictors were dropped according to the White et al.'s proposed rule of thumb. In this rule, the number of imputations used was matched to the proportion of missing data [14]. We applied the "the missing completely at random" (MCAR) procedure for the missing data to define missing mechanisms of variables for either dropping or imputing before performing multiple imputation for the cases and columns $<30 \%$ [15]. The hypothesis of MCAR was rejected at the 0.05 level by the normality test; therefore, dropping the missing observations would produce biased estimates. We imputed the missing observations 20 times. We also generated a complete dataset by aggregating the set of twenty imputations to the medians [16].

Statistical analysis Univariate and logistic regression analyses were done to identify predictors for mortality. The data was obtained on the day that the antibiotics were started. Parametric and non-parametric data were differentiated from quantitative data (continuous variables). In univariate analysis, the differences between the groups of mortality were examined using Student's $t$ test for parametric and MannWhitney $U$ test for non-parametric tests. Consultation within the study working group was used when collinearity was suspected to select which variable to retain on the basis of perceived clinical value, reliability of measurement, and availability. Backward Wald method was used for binary logistic regression analysis. $p<0.05$ was accepted as significant for further analyses. Using consultation within the study working group, the APACHE score was excluded from the regression analysis as the potential source of collinearity.

The parameters we included in univariate analyses at the start of antibiotics were the following: patient characteristics, underlying comorbid conditions and invasive procedures, clinical signs and findings, radiological data, antibiotic susceptibility data and categories (MDR, XDR), and the antibiotics used.

\section{Results}

A total of 245 patients were enrolled in the study. We excluded 61 cases with missing follow-up data and 7 more patients that did not meet microbiological requirements (missing data $>$ 30\%). Hence, we included 177 cases. The median antibiotic use period of the patients was (IQR) 13 (9-19.25) days. A 
Table 1 Risk factors for acquisition of VAP due to Acinetobacter spp.

\begin{tabular}{ll}
\hline Variable & $N=177$ \\
Underlying comorbidities & $n(\%)$ \\
Hypertension & $70(39.5)$ \\
COPD & $50(28.2)$ \\
Cerebrovascular disease & $43(24.3)$ \\
Diabetes mellitus & $41(23.2)$ \\
Congestive heart failure & $40(22.6)$ \\
Acute renal failure & $34(19.2)$ \\
Coronary artery disease & $34(19.2)$ \\
Surgery & $28(15.8)$ \\
Malignancy & $27(15.3)$ \\
Trauma & $21(11.9)$ \\
Chronic renal failure & $11(6.2)$ \\
Immunosupressive treatment & $10(5.6)$ \\
Chronic liver disease & $4(2.3)$ \\
Splenectomy & $3(1.7)$ \\
Neutropenia & $2(1.1)$ \\
Burn & $1(0.6)$ \\
HIV infection & $1(0.6)$ \\
Connective tissue disorder & $1(0.6)$ \\
Invasive procedures & $n(\%)$ \\
CVC & $146(82.5)$ \\
• Internal jugular & $75(51.4)$ \\
• Subclavian & $54(37.0)$ \\
• Femoral & $17(11.6)$ \\
Narinary catheter & $175(98.9)$ \\
Tracheostomy & $131(74.0)$ \\
Drainage catheter & $43(24.3)$ \\
& $21(11.9)$ \\
\hline
\end{tabular}

$V A P$, ventilator-associated pneumonia; $C O P D$, chronic obstructive pulmonary disease; $H I V$, human immunodeficiency virus; $C V C$, central venous catheter

total of 8 missing values $(4.52 \%)$ of creatinine met the MCAR assumptions.

I. Initial assessment (a) Patient characteristics: The median (IQR) age of the patients was 68 (52.5-79) years; 120 patients $(67.8 \%)$ were males. Underlying comorbid conditions and invasive procedures of the patients are presented in Table 1.

(b) Start of antimicrobial therapy: The median (IQR) time period between mechanical ventilation and start of antibiotics was $6(2-12)$ days.

(c) Colistin use: Colistimethate sodium (CMS) was the available formulation in the market during the study period and was used when necessary. The details of CMS use at the start of therapy are presented in Fig. 1. All patients were treated with standard antibiotic dosages (https://www.sanfordguide.com/).

II. The assessment of initial culture results

Acinetobacter spp. were recovered in blood cultures in $23(13.0 \%)$ patients, in ETA of 155 (87.6\%) cases, and BAL in 26 (14.7\%). Multiple cultures were positive for Acinetobacter spp. in $37(21 \%)$ patients. The AST results of Acinetobacter spp. are presented in Table 2. In total, 136 MDR (76.8\%), 38 XDR (21.5\%), 1 PDR, and 2 susceptible strains were recovered. When the patients were evaluated according to AST data, 69 (39\%) initially received appropriate empirical antimicrobial treatment.

III. Overall assessment (28th day of appropriate antimicrobial treatment)

(a) Outcome: On the 28th day of follow-up assessment, 89 (50.3\%) patients had died. The median time to death (IQR) was 10 (7-16) days.

(b) Drug modification: During the 28-day period, antibiotics were modified in 14 patients on the 3rd day of assessment and it was modified on the 7 th day in 16 patients. In 3 cases, modification was made in both timings reaching

Fig. 1 Colistin use at the start of therapy

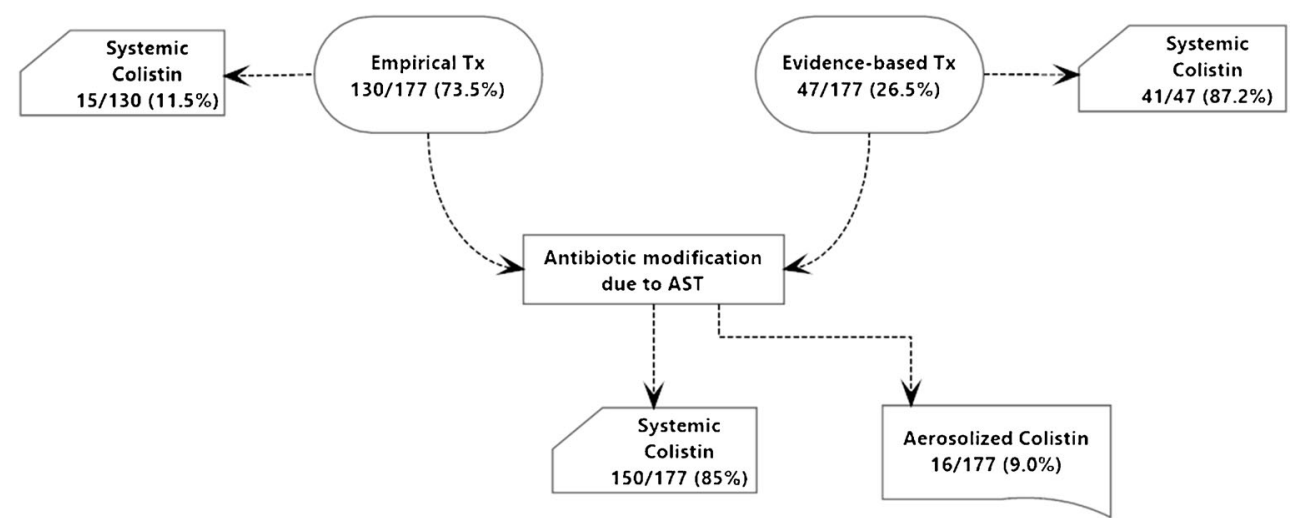


Table 2 Antimicrobial susceptibility data of 177 Acinetobacter spp. isolates

\begin{tabular}{ll}
\hline Antibiotics $(n)$ & Resistant (\%) \\
\hline Colistin (175) & $2(1.1)$ \\
Tigecycline (104) & $40(38.5)$ \\
Amikacin (174) & $143(82.2)$ \\
Gentamicin (173) & $150(86.7)$ \\
Imipenem (175) & $171(97.7)$ \\
Meropenem (175) & $172(92.3)$ \\
Piperacillin-tazobactam (160) & $159(99.4)$ \\
Cefoperazone-sulbactam (141) & $130(92.2)$ \\
Ampicillin-sulbactam (160) & $156(97.5)$ \\
Trimethoprim sulfamethoxazole (173) & $147(85.0)$ \\
Ciprofloxacin (177) & $172(97.2)$ \\
\hline
\end{tabular}

a sum of 27 on the whole. Hence, crude mortality was $48 \%(n=72)$ in patients without modification and it was $63 \%(n=17)$ with modification. (c) Antibiotic dosing: During the 28-day period, a CMS loading dose $(300 \mathrm{mg})$ was given in 21 of $150(14 \%)$. Renal dose adjustment was made in 23 patients on the 3 rd day of assessment while redosing was made on the 7th day in 15 patients. In 5 patients, dose adjustment was made in both timings reaching a total of 38 . Hence, crude mortality was $45.8 \%(n=66)$ in patients without adjustment and it was $70 \%(n=23)$ with modification.

(d) Prognostic assessment: Table 3 shows the parameters associated with mortality in univariate analyses and final logistic regression model. Consequently, appropriate empiric antimicrobial treatment (OR, 0.445 (95\% CI $0.216-0.914) ; p=0.027$ ), surgical operations performed in the last month (OR, 0.137 (95\% CI 0.037-0.499); $p=0.003$ ), fever (OR, 0.663 (95\% CI: 0.454-0.967); $p=0.033$ ), creatinine levels (OR, 1.84 (95\% CI 1.279-2.657); $p=0.001$ ), malignancy (OR, 7.095 (95\% CI 2.142-23.500); $p=$ 0.001 ), and congestive heart failure (OR, 2.341 (95\% CI 1.046-5.239); $p=0.038$ ) at the start of
Table 3 Outcome analysis of 177 patients with VAP due to Acinetobacter spp.

\begin{tabular}{|c|c|c|c|c|}
\hline \multicolumn{5}{|l|}{ Univariate analyses, significant parameters } \\
\hline & Death & Survival & Total & $p$ value \\
\hline Diabetes mellitus & $27(30.3 \%)$ & $14(15.9 \%)$ & $41(23.2 \%)$ & $0.032 *$ \\
\hline Malignant diseases & $19(21.3 \%)$ & $8(9.1 \%)$ & $27(15.3 \%)$ & $0.035^{*}$ \\
\hline Congestive heart failure & $26(29.2 \%)$ & $14(15.9 \%)$ & $40(22.6 \%)$ & $0.047 *$ \\
\hline Trauma & $3(3.4 \%)$ & $18(20.5 \%)$ & $21(11.9 \%)$ & $<0.001^{*}$ \\
\hline $\begin{array}{l}\text { Ciprofloxacin-resistant } \\
\text { Acinetobacter }\end{array}$ & $0(0.0 \%)$ & $5(5.7 \%)$ & $5(2.8 \%)$ & $0.029 *$ \\
\hline Acute renal failure & $22(24.7 \%)$ & $12(13.6 \%)$ & $34(19.2 \%)$ & $0.085^{*}$ \\
\hline Hypertension & $41(46.1 \%)$ & $29(33.0 \%)$ & $70(39.5 \%)$ & $0.091 *$ \\
\hline Surgical operation in the last month & $6(6.7 \%)$ & $22(25.0 \%)$ & $28(15.8 \%)$ & $0.001 *$ \\
\hline Judicious treatment (empirical) & $29(32.6 \%)$ & $40(45.5 \%)$ & $69(39.0 \%)$ & $0.091 *$ \\
\hline APACHE-II & $24(6-66)$ & $18(1-45)$ & $21(1-66)$ & $<0.001 * *$ \\
\hline \multicolumn{5}{|l|}{ Median (min-max) } \\
\hline Creatinine value & 1.10 & 0.70 & 0.80 & $<0.001 * *$ \\
\hline Median (min-max) & $(0.10-5.60)$ & $(0.18-5.60)$ & $(0.10-5.60)$ & \\
\hline Fever & 37.8 & 38.3 & 38.0 & $0.004 * *$ \\
\hline Median (min-max) & $(35.7-40.2)$ & $(36.0-40.5)$ & $(35.7-40.5)$ & \\
\hline \multicolumn{5}{|l|}{ Logistic regression analysis } \\
\hline & & & \multicolumn{2}{|c|}{ 95\% C.I. for $\operatorname{EXP}(B)$} \\
\hline & Sig. & OR & Lower & Upper \\
\hline Creatinine & 0.001 & 1.843 & 1.279 & 2.657 \\
\hline Fever & 0.033 & 0.663 & 0.454 & 0.967 \\
\hline Malignant diseases & 0.001 & 7.095 & 2.142 & 23.500 \\
\hline Congestive heart failure & 0.038 & 2.341 & 1.046 & 5.239 \\
\hline Judicious treatment (empirical) & 0.027 & 0.445 & 0.216 & 0.914 \\
\hline Surgical operation in the last month & 0.003 & 0.137 & 0.037 & 0.499 \\
\hline Constant & 0.040 & $3,071,378.735$ & & \\
\hline
\end{tabular}

$V A P$, ventilator-associated pneumonia

*Fisher's exact test, **Mann-Whitney $U$ 
Fig. 2 Therapeutic courses in VAP due to Acinetobacter spp.

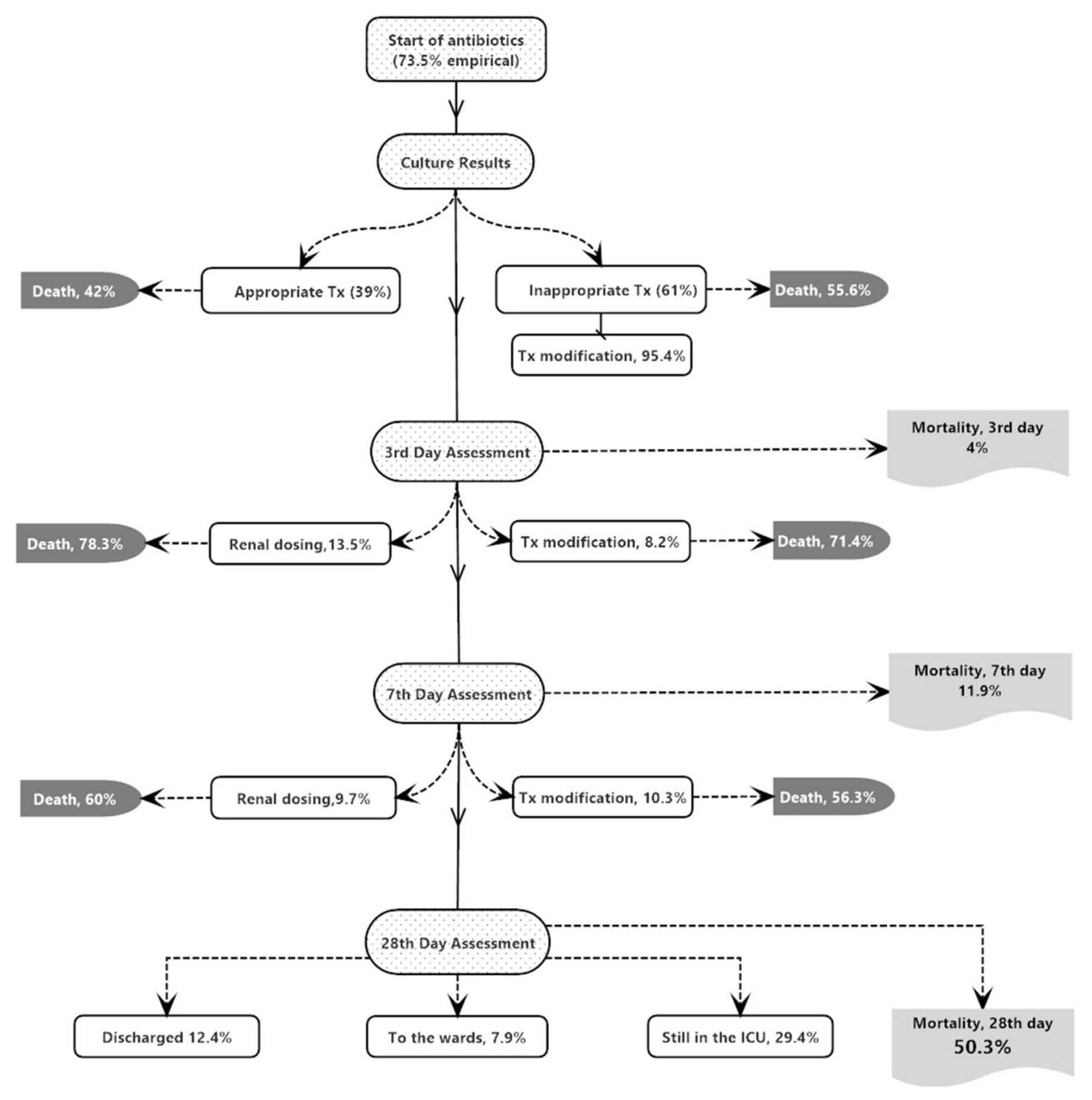

antibiotics were significantly associated with mortality. The course of anti-infective treatment is presented in Fig. 2.

\section{Discussion}

Infections due to Acinetobacter spp., particularly VAP in the ICUs, are mostly seen in critically ill or debilitated patients [17]. After 1 month of follow-up, half of the cases with VAP due to Acinetobacter spp. died in this study. The magnitude of the problem indicates the need for optimizing the diagnosis and therapy of these infections. We report that therapeutic options were quite limited and involved serious toxicities for patients with VAP due to Acinetobacter spp. In addition, the use of appropriate empirical antimicrobial therapy contributed to survival of patients along with recent surgery and fever. We hypothesize that a high fever favors a robust immunity, and surgical operation in the last month as an acute disorder without permanent chronic conditions. In contrast, renal insufficiency indicated by high creatinine levels considering the nephrotoxic potential of CMS, the backbone of therapy, or comorbid conditions like congestive heart failure and malignancy significantly contributed to a poor prognosis.

Although carbapenem resistance differs throughout the world being less common in high-income countries [8], more than $95 \%$ of Acinetobacter isolates were resistant to carbapenems in our study. Interestingly, although inappropriate antibiotic treatment contributed to mortality, we could not show a difference between XDR and MDR strains for survival. CMS seemed to be the major option in the management of the disease followed by tigecycline as a potential alternative. When carbapenem resistance exceeds $20 \%$ in a given community, then empiric CMS combination with a carbapenem (other than ertapenem), tigecycline, or sulbactam is advocated [18]. However, tigecycline has limitations due to low plasma levels limiting its use in bacteremias [19]. Accordingly, the use of tigecycline in VAP was shown to be hampered in a phase III randomized controlled trial disclosing higher mortality in the tigecycline arm [20]. Under these peculiar circumstances, what should the treating clinicians do when our data are considered? Should CMS-based regimens be given to all VAP patients as empirical treatment? This controversy is also 
reflected in our study since the physicians prescribed CMSbased regimens empirically in $11.5 \%$ of the cases while CMS was prescribed in $87.2 \%$ of the patients following AST results. Although we have disclosed that appropriate treatment contributed to survival in this study, empirical CMS use will surely result in the rapid loss of the unique option. No concrete answer seemingly exists for this dilemma even in the guidelines [21]. One potential resolution may be to use rapid diagnostic tests (RDT). Cultures were taken after the start of antibiotics in $9 \%$ of our cases and it took a median of 2 days to yield AST data. These obvious delays indicate the necessity of RDTs in routine medical practice like multiplex real-time PCR or MALDI-TOF MS [18]. Considering the limitations and since most RDTs have not been validated for respiratory secretions, they should be better used together with conventional culture systems. Furthermore, antibiotic stewardship can improve survival $[18,22]$.

The treatment of VAP due to Acinetobacter spp. is a real thorny road. When the initial antibiotic therapies were evaluated according to subsequent AST data, three-fifths of the patients did not receive appropriate empiric antimicrobial treatment. After the modification of therapy after the availability of AST results, problems related to drug side effects and clinical worsening arose. In fact, patients were prone to two types of drug modification: one due to AST results, and the second due to drug side effects. Hence, VAP prevention should become a core measure and VAP prevention bundles should be implemented [23]. Unfortunately, one-third of centers from low-middle-income countries do not have these bundles in use [24].

The strengths of our study were that we excluded polymicrobial VAP and patients with coexistent infections to limit the confounders. This is also one of the largest prospective multicenter studies evaluating VAP due to Acinetobacter. Additionally, we only included the first VAP episode. Despite the strengths, we had limitations. First, we did not have the minimum inhibitor concentration values of all infecting Acinetobacter strains. Second, there could be propensity bias as treatment of patients was not randomized. Third, since the study had an observational design, it was not possible to reach a sufficient number of cases in order to analyze each independent factor that could affect the course of Acinetobacter-induced VAP. Appropriate empiric antimicrobial treatment, immunocompetency, and comorbid conditions affected the outcome. Surveillance of antimicrobial resistance, antibiotic stewardship, VAP prevention, diagnostic improvement, and close patient follow-up appear to have paramount importance in managing CAP patients.

Acknowledgments Infectious Diseases and Clinical Microbiology Specialty Society of Turkey (EKMUD) provided the web infrastructure of the study for data submission.

\section{Compliance with ethical standards}

Conflict of interest The authors declare that they have no conflict of interest.

Ethical approval Dr. Lutfi Kirdar Education and Training Hospital's Review Board in Istanbul approved the study (02/01/2014-VIP 2014/1) and this approval was confirmed the Turkish Ministry of Health, Drugs and Pharmaceutics Agency for all participating centers. This study was performed in accordance with the Helsinki Declaration of 1964 and its later amendments.

Informed consent Yes, informed consent is obtained.

\section{References}

1. Magill SS, Edwards JR, Bamberg W, Beldavs ZG, Dumyati G, Kainer MA et al (2014) Multistate point-prevalence survey of health care-associated infections. N Engl J Med 370:1198-1208. [cited 2018 6]. http://www.ncbi.nlm.nih.gov/pubmed/24670166. https://doi.org/10.1056/NEJMoa1306801

2. Erdem H, Inan A, Altindis S, Carevic B, Askarian M, Cottle L et al (2014) Surveillance, control and management of infections in intensive care units in southern Europe, Turkey and Iran - a prospective multicenter point prevalence study. J Inf Secur 68:131-140. https://doi.org/10.1016/j.jinf.2013.11.001

3. Kalil AC, Metersky ML, Klompas M, Muscedere J, Sweeney DA, Palmer LB, et al. Management of adults with hospital-acquired and ventilator-associated pneumonia: 2016 clinical practice guidelines by the Infectious Diseases Society of America and the American Thoracic Society. Clin Infect Dis 2016;63:e61-111. doi: https://doi. org/10.1093/cid/ciw353

4. Vallés J, Pobo A, García-Esquirol O, Mariscal D, Real J, Fernández R (2007) Excess ICU mortality attributable to ventilator-associated pneumonia: the role of early vs late onset. Intensive Care Med 33: 1363-1368. https://doi.org/10.1007/s00134-007-0721-0

5. Clark NM, Zhanel GG, Lynch JP (2016) Emergence of antimicrobial resistance among Acinetobacter species. Curr Opin Crit Care 22:491-499. [cited 2018 9]. http://www.ncbi.nlm.nih.gov/pubmed/ 27552304. https://doi.org/10.1097/MCC.0000000000000337

6. Erdem H, Dizbay M, Karabey S, Kaya S, Demirdal T, Koksal I, et al. (2013) Withdrawal of Staphylococcus aureus from intensive care units in Turkey. Am J Infect Control 41. doi: https://doi.org/10. 1016/j.ajic.2013.01.041

7. Asif M, Alvi IA, Rehman SU (2018) Infection and drug resistance. Dovepress. Insight into Acinetobacter baumannii: pathogenesis, global resistance, mechanisms of resistance, treatment options, and alternative modalities. 1249-60. doi: https://doi.org/10.2147/ IDR.S166750doi: 10.2147/IDR.S166750

8. Bonell A, Azarrafiy R, Huong TVL, Viet TL, Phu VD et al (2018;doi: http://fdslive.oup.com/www.oup.com/pdf/production in progress.pdf) A systematic review and meta-analysis of ventilator associated pneumonia in adults in Asia: an analysis of national income level on incidence and etiology. Clin Infect Dis. https://doi. org/10.11821/dlxb201802008

9. The European Committee on Antimicrobial Susceptibility Testing. Breakpoint tables for interpretation of MICs and zone diameters. Version 4.0. 2014

10. The European committee on antimicrobial susceptibility testing. Breakpoint tables for interpretation of MICs and zone diameters. Version 5.0 [Internet]. 2015. doi: http://www.eucast.org 
11. The European Committee on Antimicrobial Susceptibility Testing. Breakpoint tables for interpretation of MICs and zone diameters, version 6.0. 2016

12. Magiorakos A-P, Srinivasan A, Carey RB, Carmeli Y, Falagas ME, Giske CG et al (2011) Bacteria : an international expert proposal for interim standard definitions for acquired resistance. Clin Microbiol Infect 18:268-281. http://onlinelibrary.wiley.com/doi/10.1111/j. 1469-0691.2011.03570.x/full. https://doi.org/10.1111/j.1469-0691. 2011.03570.x

13. Centers for Disease Control and Prevention (2019) (Ventilator-associated pneumonia [VAP] and non-ventilator-associated pneumonia [PNEU]) event. Device-associated module. CDC;1-16

14. White IR, Royston P, Wood AM (2011) Multiple imputation using chained equations: issues and guidance for practice. Stat Med 30: 377-399. https://doi.org/10.1002/sim.4067

15. Donders ART, van der Heijden GJMG, Stijnen T, Moons KGM (2006) Review: a gentle introduction to imputation of missing values. J Clin Epidemiol 59:1087-1091. https://doi.org/10.1016/j. jclinepi.2006.01.014

16. Cag Y, Karabay O, Sipahi OR, Aksoy F, Durmus G, Batirel A et al (2018) Development and validation of a modified quick SOFA scale for risk assessment in sepsis syndrome. PLoS One 13: e0204608. https://doi.org/10.1371/journal.pone.0204608

17. Lynch JP, Zhanel GG, Clark NM (2017) Infections due to Acinetobacter baumannii in the ICU: treatment options. Semin Respir Crit Care Med 38:311-325. https://doi.org/10.1055/s0037-1599225

18. Vazquez Guillamet C, Kollef MH (2018) Acinetobacter pneumonia: improving outcomes with early identification and appropriate therapy. Clin Infect Dis 67:1455-1462. https://academic.oup.com/ cid/article/67/9/1455/4993159. https://doi.org/10.1093/cid/ciy375

19. Isler B, Doi Y, Bonomo RA, Paterson DL (2018) New treatment options against carbapenem-resistant Acinetobacter baumannii infections. Antimicrob Agents Chemother ;1-43. doi: http://aac. asm.org/lookup/doi/10.1128/AAC.01110-18 doi: https://doi.org/ 10.1128/AAC.01110-18

20. Freire AT, Melnyk V, Kim MJ, Datsenko O, Dzyublik O, Glumcher F et al (2010) Comparison of tigecycline with imipenem/cilastatin for the treatment of hospital-acquired pneumonia. Diagn Microbiol Infect Dis 68:140-151. https://doi.org/10.1016/j.diagmicrobio. 2010.05.012

21. Tsuji BT, Pogue JM, Zavascki AP, Paul M, Daikos GL, Forrest A et al (2019) International consensus guidelines for the optimal use of the polymyxins: endorsed by the ACCP, ESCMID, IDSA, ISAP, SCCM, and SIDP. Pharmacotherapy 39:10-39. https://doi.org/10. 1002/phar.2209

22. Huang AM, Newton D, Kunapuli A, Gandhi TN, Washer LL, Isip J et al (2013) Impact of rapid organism identification via matrixassisted laser desorption/ionization time-of-flight combined with antimicrobial stewardship team intervention in adult patients with bacteremia and candidemia. Clin Infect Dis 57:1237-1245. https:// doi.org/10.1093/cid/cit498

23. Vazquez Guillamet C, Kollef MH (2018) Is zero ventilatorassociated pneumonia achievable? Clin Chest Med 39:809-822. [cited 2018 6]. http://www.ncbi.nlm.nih.gov/pubmed/30390751. https://doi.org/10.1016/j.ccm.2018.08.004

24. Alp E, Cookson B, Erdem H, Rello J, Akhvlediani T, Akkoyunlu Y et al (2019) Infection control bundles in intensive care: an international cross-sectional survey in low-middle income countries. J Hosp Infect 101:248-256. https://doi.org/10.1016/j.jhin.2018.07. 022

Publisher's note Springer Nature remains neutral with regard to jurisdictional claims in published maps and institutional affiliations.

\section{Affiliations}

Hakan Erdem ${ }^{1}$ (D) Yasemin Cag $^{2} \cdot$ Serap Gencer ${ }^{3}$. Serhat Uysal ${ }^{4} \cdot$ Zuhal Karakurt $^{5} \cdot$ Rezan Harman $^{6} \cdot$ Emel Aslan $^{7}$. Esmeray Mutlu-Yilmaz ${ }^{8}$. Oguz Karabay ${ }^{9}$ - Yesim Uygun ${ }^{10} \cdot$ Mehmet Ulug $^{11} \cdot$ Selma Tosun ${ }^{12}$ - Arzu Dogru ${ }^{2}$. Alper Sener ${ }^{13} \cdot$ Mustafa Dogan ${ }^{14} \cdot$ Rodrigo Hasbun $^{15} \cdot$ Gul Durmus $^{16} \cdot$ Hale Turan $^{17}$. Ayse Batirel ${ }^{18} \cdot$ Fazilet Duygu $^{19}$. Asuman Inan ${ }^{20}$. Yasemin Akkoyunlu ${ }^{21} \cdot$ Guven Celebi $^{22}$. Gulden Ersoz ${ }^{23} \cdot$ Tumer Guven $^{24} \cdot$ Ozgur Dagli $^{16}$. Selma Guler ${ }^{25}$ - Meliha Meric-Koc ${ }^{21} \cdot$ Serkan Oncu ${ }^{26}$ • Jordi Rello ${ }^{27}$

1 IDI-IRI, Ankara, Turkey

2 Goztepe Training and Research Hospital, Department of Infectious Diseases and Clinical Microbiology, Medeniyet University School of Medicine, Istanbul, Turkey

3 Department of Infectious Diseases and Clinical Microbiology, Acibadem Maslak Hospital, Istanbul, Turkey

4 Kanuni Research and Training Hospital, Department of Infectious Diseases and Clinical Microbiology, University of Health Sciences, Trabzon, Turkey

5 Respiratory Intensive Care Unit, Sureyyapasa Chest Diseases and Thoracic Surgery Education and Research Hospital, Istanbul, Turkey

6 Department of Infectious Diseases and Clinical Microbiology, Toros State Hospital, Mersin, Turkey
7 Department of Infectious Diseases and Clinical Microbiology, Dicle University School of Medicine, Diyarbakir, Turkey

8 Department of Infectious Diseases and Clinical Microbiology, Samsun Training and Research Hospital, Samsun, Turkey

9 Department of Infectious Diseases and Clinical Microbiology, Sakarya University School of Medicine, Sakarya, Turkey

10 Department of Infectious Diseases and Clinical Microbiology, Kosuyolu Training and Research Hospital, Istanbul, Turkey

11 Department of Infectious Diseases and Clinical Microbiology, Private Umit Hospital, Eskisehir, Turkey

12 Department of Infectious Diseases and Clinical Microbiology, Izmir Bozyaka Training and Research Hospital, Izmir, Turkey

13 Department of Infectious Diseases and Clinical Microbiology, Onsekiz Mart University School of Medicine, Canakkale, Turkey 
14 Department of Infectious Diseases and Clinical Microbiology, Namik Kemal University School of Medicine, Tekirdag, Turkey

15 Department of Infectious Diseases, UT Health McGovern Medical School, Houston, TX, USA

16 Bursa Yuksek Ihtisas Research and Training Hospital, Department of Infectious Diseases and Clinical Microbiology, University of Health Sciences, Bursa, Turkey

17 Department of Infectious Diseases and Clinical Microbiology, Baskent University School of Medicine, Konya, Turkey

18 Dr. Lutfi Kirdar Training and Research Hospital, Department of Infectious Diseases and Clinical Microbiology, University of Health Sciences, Istanbul, Turkey

19 Department of Infectious Diseases and Clinical Microbiology, Ankara Oncology Training and Research Hospital, Ankara, Turkey Department of Infectious Diseases and Clinical Microbiology, Haydarpasa Numune Training and Research Hospital, Istanbul, Turkey
21 School of Medicine, Department of Infectious Diseases and Clinical Microbiology, Bezmialem Vakif University, Istanbul, Turkey

22 Faculty of Medicine, Department of Infectious Diseases and Clinical Microbiology, Bulent Ecevit University,

Zonguldak, Turkey

23 Faculty of Medicine, Department of Infectious Diseases and Clinical Microbiology, Mersin University, Mersin, Turkey

24 Department of Infectious Diseases and Clinical Microbiology, Karadeniz Eregli Medikal Park Hospital, Eregli, Turkey

25 Department of Infectious Diseases and Clinical Microbiology, Kahramanmaras Nezip Fazil State Hospital, Kahramanmaras, Turkey

26 School of Medicine, Department of Infectious Diseases and Clinical Microbiology, Adnan Menderes University, Aydin, Turkey

27 Vall d'Hebron Institute of Research, CIBERES, Barcelona, Spain 